\title{
Self-assembly of Nanometer-scale Magnetic Dots with Narrow Size Distributions on an Insulating Substrate
}

\author{
Zheng Gai, ${ }^{1,2}$ Biao Wu, ${ }^{3,1}$ J.P. Pierce, ${ }^{4,1}$ G.A. Farnan, ${ }^{1}$ Dajun Shu, ${ }^{5,6}$ Mu Wang, ${ }^{6,5}$ Zhenyu Zhang, ${ }^{1,5}$ and Jian Shen ${ }^{1}$ \\ ${ }^{1}$ Solid State Division, Oak Ridge National Laboratory, Oak Ridge, TN 37831 \\ ${ }^{2}$ Department of Physics, Peking University, Beijing, 100871, P.R. China \\ ${ }^{3}$ Department of Physics, The University of Texas, Austin, Texas 78712 \\ ${ }^{4}$ Department of Physics and Astronomy, University of Tennessee, Knoxville, Tennessee 37996 \\ ${ }^{5}$ International Center for Quantum Structures, Chinese Academy of Sciences, Beijing 100080, P.R. China \\ ${ }^{6}$ Department of Physics, Nanjing University, Nanjing, 210093, P.R. China
}

(Dated: October 29, 2018)

\begin{abstract}
The self-assembly of iron dots on the insulating surface of $\mathrm{NaCl}(001)$ is investigated experimentally and theoretically. Under proper growth conditions, nanometer-scale magnetic iron dots with remarkably narrow size distributions can be achieved in the absence of a wetting layer. Furthermore, both the vertical and lateral sizes of the dots can be tuned with the iron dosage without introducing apparent size broadening, even though the clustering is clearly in the strong coarsening regime. These observations are interpreted using a phenomenological mean-field theory, in which a coverage-dependent optimal dot size is selected by strain-mediated dot-dot interactions.
\end{abstract}

PACS numbers: 81.07.Ta,61.46.+w,68.37.Ps,68.35.Md

Clustering on surfaces by nucleation and growth during atom deposition has been an important subject in basic and applied science for decades [1], 2]. Recent efforts have been focused on searching for methods to obtain nanometer-scale clusters with narrow size distributions. Such clusters or quantum dots are potentially valuable for optical, electronic, and magnetic device applications, but mass production of such structures by lithography or etching-based fabrication has proved to be exceptionally challenging 13, 4. Alternatively, it has been realized that the strain energy associated with the lattice mismatch between the dot and the substrate materials can be exploited to induce self-assembled formation of quantum dots with narrow size distributions. This has generated much excitement, particularly in the area of semiconductor quantum dots [3, 4, 5, 6, 7, 8]. In such cases, the growth of the dots often proceeds in the StranskiKrastanow (SK) mode, which is characterized by the presence of a wetting layer prior to three-dimensional (3D) clustering. To date, the precise mechanism for size selection in semiconductor quantum dot systems remains a subject of active debate [4, 5, 6, 9]: some attribute them to strain-induced thermodynamic equilibrium states, while others associate them with metastable configurations due to kinetic limitations.

Although improved size uniformity can be achieved in quantum dot formation via the SK growth mode, the presence of a wetting layer is often undesirable, particularly for electronic and magnetic device applications of metallic/magnetic quantum dots. For this reason, it is preferred to fabricate quantum dots in the VolmerWeber (VW) growth mode, which is characterized by immediate 3D clustering on the substrate surface. Indeed, considerable recent efforts have been devoted to metallic/magnetic quantum dot formation on various substrates [10, 11, 12], but no significant size uniformity has been achieved in such studies.

In this Letter we investigate the self-assembly of iron dots on $\mathrm{NaCl}(001)$, an insulating substrate, by thermal deposition and variable-temperature atomic force microscopy in ultrahigh vacuum. We show that, by properly choosing the growth conditions, nanometer-scale magnetic iron dots with remarkably narrow size distributions can be achieved in the absence of a wetting layer (VW growth). Moreover, by changing the dosage of iron, we can tune both the vertical and lateral sizes of the dots without introducing apparent size broadening, even though the clustering is already in the strong coarsening regime, signified by the decrease in dot density as a function of the iron dosage. The preserved narrowness in the island size distributions is in clear contradiction with the expectations of existing understanding of clustering on surfaces in the coarsening regime [2]. We interpret these observations within a phenomenological mean-field theory, in which a coverage-dependent optimal dot size is selected by the competition between the self-energy of a dot, i.e. the total energy of an isolated $\mathrm{Fe}$ dot on $\mathrm{NaCl}(100)$, and the energy of the strain-induced dipolar interaction between the dots.

The experiments were performed in an ultrahigh vacuum (UHV) system with base pressure of $1 \times 10^{10}$ torr. The system is equipped with electron beam sources, laser molecular beam epitaxy, and an in-situ Omicron variable-temperature UHV beam deflection atomic force microscope (AFM)/ scanning tunneling microscope (STM) with cooling and heating facilities covering a temperature range of (13-1500) K. The noncontact mode AFM was used to study the surface morphology in this work. The $\mathrm{NaCl}$ single crystal substrates were cleaved in air, then were immediately loaded into the UHV cham- 
ber and were annealed to $530 \mathrm{~K}$ for one hour to remove surface contamination prior to the experiments. AFM images of the cleaved $\mathrm{NaCl}(001)$ surface show monatomic height steps and large terraces with no detectable adsorbates. The iron was evaporated from an Fe wire $(5 \mathrm{~N}$ purity) heated by electron beam bombardment at a rate of $0.04 \mathrm{ML} / \mathrm{min}$ (1ML is equivalent to the nominal surface atomic density of bcc $\mathrm{Fe}(110), 1.7 \times 10^{15}$ atoms $/ \mathrm{cm}^{2}$ ). The iron dots can be formed only within a finite temperature window, since it is known that the morphology of the substrate changes dramatically if the temperature is above $720 \mathrm{~K}[13$, whereas at low temperatures, only random clusters or percolated iron films form 14 . The dots discussed in this Letter are grown at a substrate temperature of $530 \mathrm{~K}$.
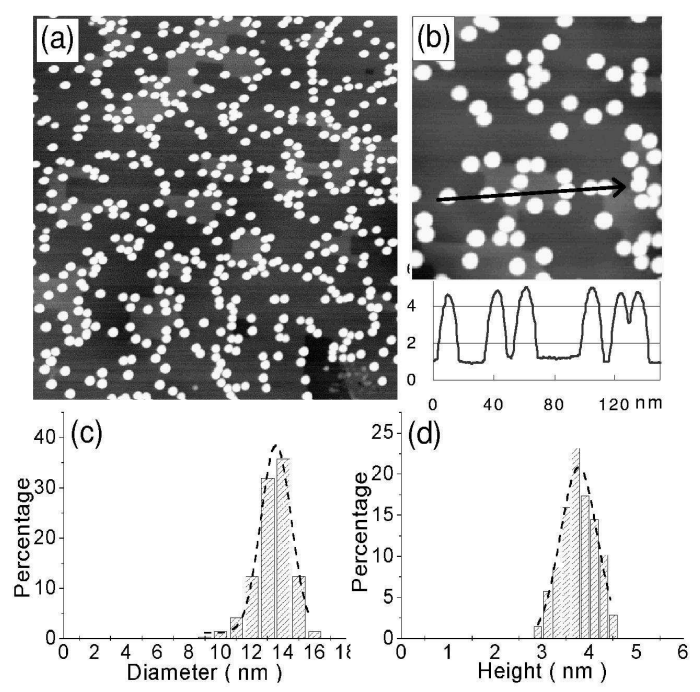

FIG. 1: Nanometer-scale iron dots grown on $\mathrm{NaCl}(001)$ with a nominal Fe dose of $1.7 \mathrm{ML}$. (a) A typical NC-AFM image. Scan area $500 \mathrm{~nm} \times 500 \mathrm{~nm}$. (b) Close up and line profile (150 $\mathrm{nm}$ long) of the dots. Scan area $200 \mathrm{~nm} \times 200 \mathrm{~nm}$. The unit in the line profile is $\mathrm{nm}$. (c) and (d) Diameter and height distributions of the dots shown in (a) and the corresponding Gaussian fits. The center and dispersion for the diameter distribution are $13.5 \mathrm{~nm}$ and $1.1 \mathrm{~nm}$, respectively; and for the height distribution are $3.76 \mathrm{~nm}$ and $0.36 \mathrm{~nm}$, respectively.

Nanometer-scale dots can be formed directly on the $\mathrm{NaCl}(001)$ surface without a wetting layer, indicating that the growth proceeds in the Volmer-Weber mode. As shown in the AFM images in Figs. 1 and 2, deposited Fe atoms tend to nucleate and form dots even at submonolayer coverage. The preferred VW growth mode is not too surprising, given the large surface free energy difference $\left(2.48 \mathrm{~J} / \mathrm{m}^{2}\right.$ for Fe versus $0.18 \mathrm{~J} / \mathrm{m}^{2}$ for $\left.\mathrm{NaCl}\right)$ [15] and the large lattice mismatch between $\mathrm{Fe}$ and $\mathrm{NaCl}[16]$. The most eye-catching characteristic of the dots is their size uniformity. Fig. 1 (a) shows a representative AFM image of the dots with a nominal Fe thickness of 1.7ML. The very uniform dots are randomly distributed on the terraces. Fig. 1 (b) is a close up together with a line profile of the dots. Estimated from the line scan, the dots are 3.5 to $4 \mathrm{~nm}$ in height, and around $14 \mathrm{~nm}$ in diameter [17. Fig. 1 (c) and (d) are the lateral size and height distributions of the dots shown in Fig.1 (a) along with the corresponding Gaussian fits. Consistent with the line profile, the centers of the Gaussian fits of the height and diameter distributions of the dots appear at $3.76 \mathrm{~nm}$ and $13.5 \mathrm{~nm}$, respectively. Each distribution has a very narrow width. The dispersions, $\Delta h=\left(\left\langle h^{2}\right\rangle-\langle h\rangle^{2}\right)^{1 / 2}$, of the height and diameter distributions are only $0.36 \mathrm{~nm}$ and $1.1 \mathrm{~nm}$, respectively, which are less than $10 \%$ of the average height and diameter values. Such narrow dispersions are comparable with the narrowest dispersions achieved in semiconductor quantum dot growth via the SK mode [8], and are particularly striking because clusters grown via the VW mode normally have much broader distributions [3, 10, 11, 12].
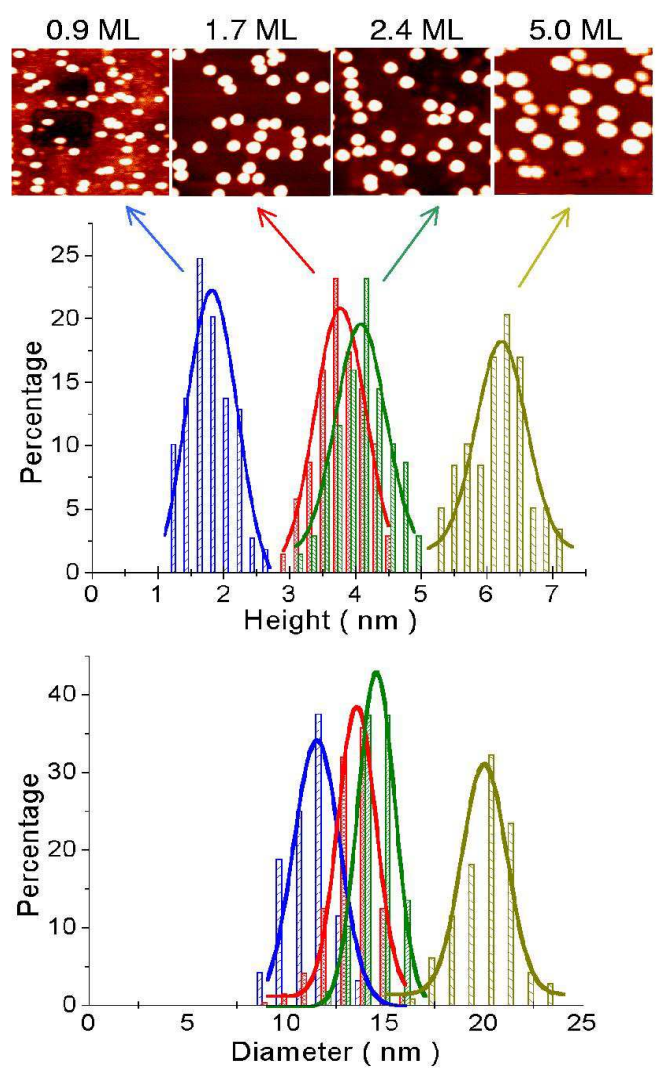

FIG. 2: Typical NC-AFM images and height and diameter distributions for dots grown on $\mathrm{NaCl}(001)$ at different Fe coverages $\theta$. The image area for each coverage is $150 \mathrm{~nm} \times 150$ $\mathrm{nm}$. The dispersions for the height distributions for the four coverages are all $0.36 \mathrm{~nm}$; and the dispersions for the diameter distributions are $0.9 \mathrm{~nm}, 1.1 \mathrm{~nm}, 1.1 \mathrm{~nm}$, and $1.2 \mathrm{~nm}$, for the four coverages, respectively.

Another striking observation lies in the coverage dependence of the dots shown in Fig. 2. As the Fe coverage 
increases, the dots grow larger and taller, but an optimal size of the dots is always selected while the narrowness of their lateral and vertical size distributions is preserved. Our quantitative analysis of the dispersions does not yield any significant additional (coverage-dependent) broadening of the dot size distributions, consistent with the impression drawn by naked eye from the AFM images shown in Fig. 2. In Fig. 3, the optimal height and lateral size of the dots are plotted as functions of the iron coverage, together with the density of the dots. The decrease of the dot density with increasing coverage indicates that the growth of the dots is clearly in the coarsening regime. This observation on the variation of the island density makes the persistence of the narrow size distributions even more dramatic, because in the coarsening regime, the absolute size distribution of the islands is expected to broaden with increasing coverage [2].

Preliminary surface magneto-optical Kerr effect (SMOKE) measurements show that these iron dots are superparamagnetic in nature. The details of the magnetic investigation, however, are beyond the scope of the present paper, which focuses on the formation of the highly uniform dots. In order to understand the underlying formation mechanism of these nanoclusters, it is highly desirable to know both their detailed atomic structures and their stability. Unfortunately, the iron dots formed on $\mathrm{NaCl}$ cannot be imaged by STM with higher (atomic) resolution. On the other hand, in-situ time (from immediately after formation up to 10 days) and temperature (from room temperature up to $550 \mathrm{~K}$ ) dependent AFM studies reveal little change in the morphology of the Fe dots, indicating that the dot arrays on the surface are in an energetically favorable configuration that is at least metastable.

At the present, the precise underlying mechanism for the formation of these narrow-sized iron dots is not completely clear, but the following phenomenological meanfield theory, stressing on strain-mediated dot-dot interactions, is able to reproduce most of the salient features observed in the experiment. First, the fact that the preferred size of the Fe dots is hardly changed upon annealing clearly indicates that the energy per atom $E$, equivalently, the chemical potential, of the Fe dot assembly must have a local minimum at the observed optimal size for a given coverage. This suggests that the qualitative form of the energy $E$ follows the generic behavior shown in Fig. 4 for a given coverage: When the volume approaches zero, $E$ is close to the energy of one adatom; when the dot grows very large, $E$ is about the energy per atom in a bulk Fe crystal, which is much smaller than the energy of one adatom. Between the two limits there exists a local minimum, mainly induced by the elastic relaxation caused by the discontinuity of the intrinsic surface stress tensor at the dot edges [A]. Secondly, the observation of different optimal sizes at different coverages shown in Fig. 2 suggests that the local energy minimum
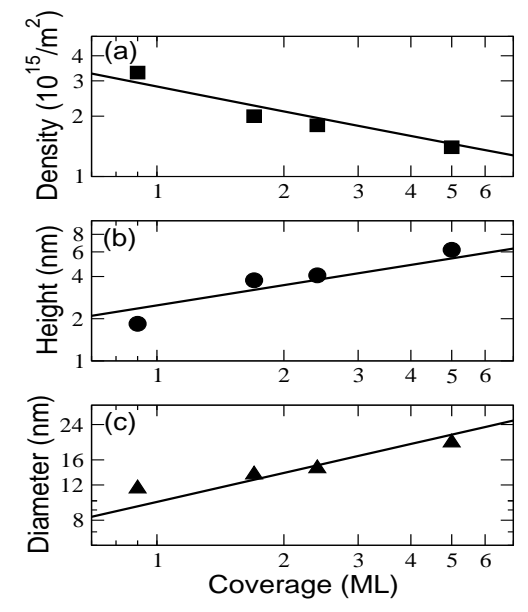

FIG. 3: The density (a), the peak height (b), and the peak diameter (c) of the iron dots as functions of the Fe coverage obtained from the Gaussian fits, in comparison with the mean field theory predictions (straight lines). Only a single parameter, $\alpha=0.46$, is used to fit all the slopes of the three independent plots.

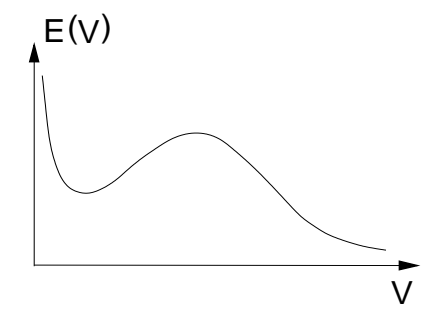

FIG. 4: Schematic drawing of the energy per atom for the Fe dot assembly as a function of the volume $V$ at a given coverage, showing the existence of local energy minimum.

is a function of the iron coverage, implying that dot-dot interactions should contribute substantially to the energy $E$. Based on these considerations, we can express $E$ as a function of both the iron coverage $\theta$ and the dot size $V$ within the mean field approximation as

$$
E(\theta, V)=E_{0}(V)+P(\theta, V),
$$

where $E_{0}$ is the self-energy of an isolated dot and $P$ is the dot-dot interaction energy.

The interaction energy per atom, $P(\theta, V)$, is originated from misfit-induced strain within the system. By adopting the standard dipole-dipole interaction between two dots as $P\left(V_{1}, V_{2}\right) \propto V_{1} V_{2} / r^{3}$ 迎, we have

$$
P(\theta, V)=g \frac{\theta^{3 / 2}}{V^{1 / 2}},
$$

where $g$ is a constant related to the interaction strength and the effective number of the nearest neighbors of a given dot. We note that, on one hand, the long range 
dot-dot interaction is important enough in influencing the energy per atom of the dot assembly, thereby the existence of an optimal dot size; on the other hand, it is not strong enough to cause the center of mass of a given dot to change, thereby the lacking of the spatial order among the dots 18 .

The self-energy $E_{0}(V)$ of an isolated dot of size $V$ includes all possible contributions to the dot, such as the strain energy, interface energy, step energy, and kink energy, but it does not depend on the total coverage $\theta$. In principle, an explicit, but rather complex, expression can be obtained from existing studies of strain-mediated dot formation in heteroepitaxy, such as Eq. (4.15) of Ref. (4. But surprisingly, our detailed analysis based on Eq. (4.15) [4 shows that no qualitatively correct fitting could be obtained for the range of iron coverages explored in the present experiment. In the following, we choose the simple expression

$$
E_{0}(V)=b V^{\alpha}
$$

as an alternative phenomenological approach, and show that the corresponding energy per atom is capable of explaining many of the salient features observed in the experiment.

First, by setting the first derivative of the energy per atom $E(\theta, V)$ with respect to the volume $V$ equal to zero, we obtain a coverage-dependent local energy minimum, at the volume $b \alpha V^{\alpha+1 / 2}=\frac{g}{2} \theta^{3 / 2}$. This, in turn, yields the relation between the density of the $\operatorname{dots} \theta / V$ and the coverage $\theta$

$$
b \alpha\left(\frac{\theta}{V}\right)^{-(\alpha+1 / 2)}=\frac{g}{2} \theta^{1-\alpha} .
$$

With $\alpha=0.46$, our theory gives an excellent fit to the experimental data as shown in Fig. 3(a). Next, assuming that the equilibrium shape of the dots does not change with the volume [19], we can also obtain the dot height $(h)$ and the diameter $(D)$ as functions of the coverage, given by $h \propto \theta^{0.52}$ and $D \propto \theta^{0.52}$, respectively. These two relationships also find very good fits to the experimental data as shown in Figs. 3(b) and 3(c). The fact that the exponent $\alpha$ as a single fitting parameter yields very good fittings to the slopes of three independent sets of experimental observations hints on the physical validity of the choice made in Eq. (3). Whereas the precise underlying physics for the success of Eq. (3) remains to be explored, we suspect that it could be related to the unusually large lattice mismatch in the present system [16]. Finally, we note that the dispersion of the distribution of the island heights (or the diameters) shows little change with the coverage, whereas our mean field theory predicts that the dispersion should increase with the coverage, $\Delta h \propto \theta^{0.4}$. This discrepancy is not too surprising, because within the mean-field theory, the resulting optimal size of the islands can be reliably obtained, but the exponent of the scaling function around the optimal value is typically overestimated, a trend well recognized in critical phenomena [20].

In summary, we have shown that, under proper growth conditions, the self-assembly of iron dots on the insulating surface of $\mathrm{NaCl}(001)$ leads to the formation of nanometer-scale magnetic iron dots with narrow size distributions in the absence of a wetting layer. We have also demonstrated that the vertical and lateral sizes of the dots can both be changed by the iron coverage while the narrow size distributions are preserved, even though the clustering is clearly in the strong coarsening regime. These striking observations have been interpreted successfully with a phenomenological mean-field theory.

We are grateful to E.W. Plummer for invaluable discussions. This work was supported in part by the LDRD of ORNL, managed by UT-Battelle, LLC for the USDOE ( DE-AC05-00OR22725), and by the NSF (DMR-0071893 (BW) and DMR-0105232 (JP)).

[1] J.A. Venables, Phil. Mag. 27, 697 (1973).

[2] For an excellent review of earlier works, see: M. ZinkeAllmang, L.C. Feldman, and M.H. Grabow, Surf. Sci. Rep. 16, 377 (1992).

[3] P. Moriarty, Rep. Prog. Phys. 64, 297 (2001); S. A. Wolf et al., Science, 294, 1488 (2001).

[4] V.A. Shchukin and D. Bimberg, Rev. Mod. Phys. 71, 1125 (1999) and references therein.

[5] Y.W. Mo, D.E. Savage, B.S. Swartzentruber, and M.G. Lagally, Phys. Rev. Lett. 65, 1020 (1990); J. Tersoff, C. Teichert, and M.G. Lagally, Phys. Rev. Lett. 76, 1675 (1996).

[6] D.E. Jesson, M. Kästner, and B. Voigtländer, Phys. Rev. Lett. 84, 330 (2000); F.M. Ross, R.M. Tromp, and M.C. Reuter, Science, 286, 1931 (1999).

[7] Q. Xie, A. Madhukar, P. Chen, and N.P. Kobayashi, Phys. Rev. Lett. 75, 2542 (1995).

[8] D. Leonard et al., Appl. Phys. Lett. 63, 3203 (1993); V.M. Ustinov et al., Appl. Phys. Lett. 72, 362 (1998); S.H. Xin et al., Appl. Phys. Lett. 69, 3884 (1996).

[9] I. Daruka and A.L. Barabasi, Phys. Rev. Lett. 79, 3708 (1997).

[10] O. Fruchart, M. Klaua, J. Barthel, and J. Kirschner, Phys. Rev. Lett. 83, 2769 (1999); Y. Park, S. Adenwalla, G.P. Felcher, S.D. Bader, Phys. Rev. B 52, 12779 (1995); C.T. Yu, D.Q. Li, J. Pearson, S.D. Bader, Appl. Phys. Lett. 79, 3848 (2001).

[11] L. Huang, S.J. Chey, and J.H. Weaver. Phys. Rev. Lett. 80, 4095 (1998).

[12] M. Bäumer, and H.J. Freund, Prog. Surf. Sci., 61, 127 (1999); A. Sugawara, and M.R. Scheinfein, Phys. Rev. B 56, R8499 (1997).

[13] M. Szymonski et al., Prog. Surf. Sci. 67, 123 (2001).

[14] Z. Gai, G.A. Farnan, J. Pierce, and J. Shen, to be published.

[15] F.R. de Boer et al., Cohesion in Metals (North Holland, Amsterdam, 1988); P.A. Mulheran, Modelling Simul. Mater. Sci. Eng. 2, 1123 (1994). 
[16] The lattice constants of bcc $\mathrm{Fe}$ and $\mathrm{NaCl}$ are $2.87 \AA$ and $5.46 \AA$, the nearest neighbors of bcc Fe(110) and $\mathrm{NaCl}(100)$ are separated by $2.49 \AA$ and $3.99 \AA$, respectively. The Fe dots are likely in bcc phase because its fcc phase exists only in bulk at temperatures above 1186 $\mathrm{K}$, or in Fe films grown on fcc substrates (e.g. $\mathrm{Cu}(100)$ ) whose lattice constants are very close to that of Fe.

[17] The lateral diameters of the dots are overestimated because of the tip effect. However, the lateral size can be estimated with an assumed shape of the dots in addition to the known information of height, density, and total dosages. For the 1.7 ML Fe dot, assuming a truncated pyramid shape, the average lateral size of the dots is around 7 to $8 \mathrm{~nm}$ instead of $14 \mathrm{~nm}$.

[18] F. Liu, A.H. Li, and M.G. Lagally, Phys. Rev. Lett. 87, 126103 (2001).

[19] C. Duport, C. Priester, and J. Villain, in Morphological Organization During growth and Removal, edited by Z.Y. Zhang and M.G. Lagally (World Scientific, Singapore 1998).

[20] K.G. Wilson, Rev. Mod. Phys. 55, 583 (1983). 